\title{
Служба Солнца KRIM в радиодиапазоне
}

\author{
A.Е. Вольвач, К.В. Самисько, С.А. Самисько, И.В. Якубовская \\ ФГБУН “Крымская астрофизическая обсерватория РАН”, Научный, Крым, 298409 \\ volvach@craocrimea.ru
}

Поступила в редакцию 15 ноября 2017 г.

\begin{abstract}
Аннотация. Четыре робот-радиотелескопа KрAO, объединенные в Службу Солнца KRIM, ведут наблюдения Солнца в режиме мониторинга и алертов. Радиотелескоп РТ-22 оснащен поляриметрами на длины волн $0.8,2.0,2.3,2.8$ и 3.5 см, позволяющими регистрировать интенсивность и круговую поляризацию радиоизлучения. Диаграммы направленности на указанных волнах составляют 2.5$6.0^{\prime}$. Радиотелескоп РТ-2 принимает излучение сантиметрового диапазона длин волн для получения сведений о процессах, протекающих в нижней хромосфере. Радиотелескоп РТ-3 принимает излучение дециметрового диапазона длин волн с целью сбора сведений о процессах, происходящих в верхней хромосфере и нижней короне. Радиотелескоп РТ-М принимает излучение метрового диапазона длин волн для обнаружения корпускулярных потоков, направляющихся к Земле в результате солнечных вспышек. Одновременные наблюдения на четырех инструментах, которые перекрывают диапазон длин волн от 8 мм до 1.2 м, дают возможность получать информацию для анализа процессов энерговыделения в атмосфере Солнца и краткосрочного прогноза солнечной активности. Данные радиомониторинга солнечной активности сохраняются в реальном времени в цифровом виде и выставляются на сайты мировой службы Солнца, которая включает 14 наземных станций в кооперации с орбитальными обсерваториями.

SOLAR SERVICE KRIM IN THE RADIO RANGE, by A.E. Volvach, K.V. Samis'ko, S.A. Samis'ko, I.V. Yakubouskaya. Four automated radio telescopes of CrAO integrated into the Solar Service KRIM perform solar observations in the monitoring and alert modes. The RT-22 radio telescope is equipped with polarimeters at wavelengths $0.8,2.0,2.3,2.8$ and $3.5 \mathrm{~cm}$, which allow recording the intensity and circular polarization of radio emission. The directivity diagrams at the mentioned waves are $2.5-6.0^{\prime}$. The RT-22 radio telescope receives emission at centimeter wavelengths to gain knowledge about processes in the lower chromosphere. The RT-3 receives emission at decimeter wavelengths to derive data about processes in the upper chromosphere and lower corona. The RT- $M$ receives emission at meter wavelengths to detect corpuscular fluxes directed to the Earth due to solar flares. Simultaneous observations at four telescopes which cover the wavelength range from $8 \mathrm{~mm}$ to $1.2 \mathrm{~m}$ allow us to acquire information for analyzing energy release processes in the solar atmosphere and making short-term prediction of solar activity. Data on the radio monitoring of solar activity are maintained in real time in a digital format and presented on websites of the International Solar Service, which involves 14 ground-based stations in cooperation with orbital observatories.
\end{abstract}

Ключевые слова: Солнце, вспышки, радиоизлучение 


\section{1 Введение}

Исследования активности ближайшей звезды - Солнца - имеют как фундаментальный, так и прикладной аспект. Возмущение, вызванное солнечной вспышкой, сопровождается генерацией радиоизлучения, воздействует на биосферу и влияет на качество работы многих устройств наземного и космического оборудования. Регулярные наблюдения радиоизлучения Солнца проводятся Мировой сетью станций радиослужбы Солнца. На основе этих данных ежедневно публикуется список состоявшихся геоэффективных событий и краткосрочный прогноз их возможного появления. Для получения систематических данных о состоянии солнечной активности в Крымской астрофизической обсерватории (KрAО) были сконструированы и длительное время эксплуатируются радиотелескопы сантиметрового, дециметрового и метрового диапазонов длин волн (рис. 1) (Юровский, 2013).

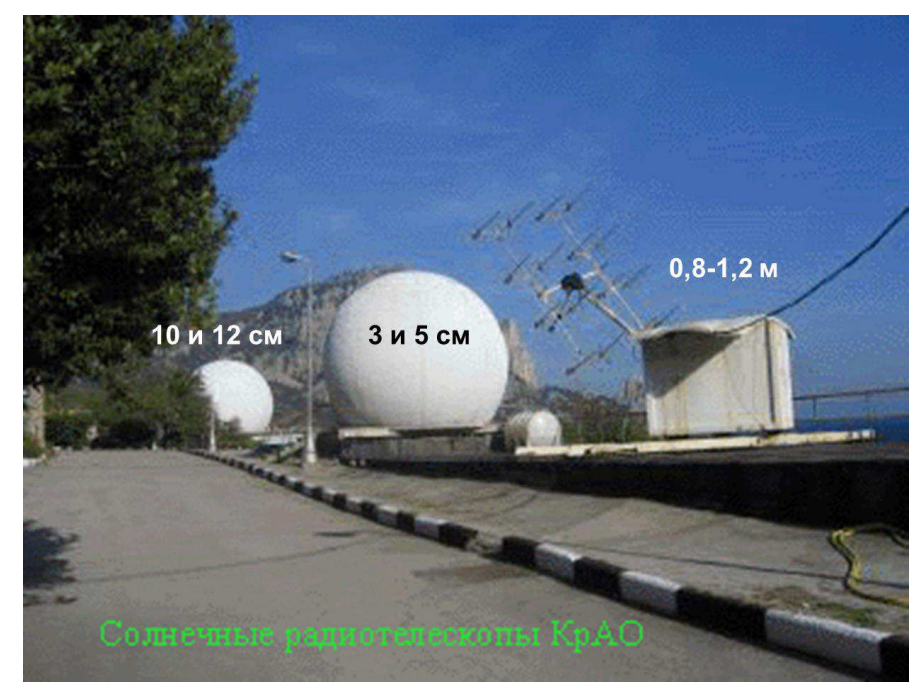

Рис. 1. Радиотелескопы РT-3, РT-2, РT-M Службы Солнца KRIM

Радиотелескоп РТ-22 КрАО оснащен радиометрическими комплексами миллиметровых и сантиметровых длин волн, с помощью которых проводятся наблюдения Солнца в последовательном варианте. Система управления РТ-22 КрАО обеспечивает точность наведения +/- 5 угл. сек. В режиме сопровождения обеспечивается коррекция координат с учетом ошибок наведения, обусловленных весовыми и температурными деформациями конструкции антенны радиотелескопа.

1. Миллиметровый комплекс. Наблюдения Солнца проводятся на двух длинах волн одновременно с регистрацией параметров Стокса (I) на длине волны 8.2 мм и (I, V) на длине волны 13.5 мм. Угловое разрешение радиотелескопа составляет 2.5 угл. мин на обеих длинах волн. По чувствительности радиометры мм-диапазона сравнимы с радиометрами см-диапазона.

2. Сантиметровый комплекс. Наблюдения Солнца выполняются с помощью диагностического комплекса, созданного на базе РТ-22 KрАО и четырехволнового поляриметра. Угловое разрешение радиотелескопа на длинах волн $3.5,2.8,2.3$ и 2.0 см составляет $6.0,5.0,4.1$ и 3.6 угл. мин соответственно. Поляриметр позволяет регистрировать полную интенсивность (I) и поляризованную по кругу составляющую (V) радиоизлучения одновременно на четырех длинах волн с наименьшим временным разрешением 0.1 с. Чувствительность радиотелескопа составляет 0.1 солнечных единиц потока.

Программное обеспечение при наблюдениях Солнца для обоих комплексов однотипно. Процесс наблюдений заключается в сканировании диска Солнца по прямому восхождению с шагом по скло- 
нению, равному 1-2 угл. мин, с последующим наведением антенны на максимум радиоизлучения локального источника на самой короткой длине волны и слежением за выбранной активной областью. Выбор объекта наблюдений осуществлялся в результате оценки прогностических параметров для каждого из локальных источников, обнаруженных на диске Солнца по результатам сканирования. Система управления РТ-22, сбора и обработки информации с выходов радиополяриметров создана на базе аппаратуры КАМАК и ЭВМ. Регистрация сигналов выполняется путем последовательного преобразования аналогового сигнала каждого из трех (восьми) НЧ-каналов поляриметрических комплексов, подаваемых на АЦП через аналоговый коммутатор с частотой коммутации 100 Гц и накоплением результата за 1 с. Обработка данных наблюдений проводится с помощью специальной программы и включает нормировку, учет паразитных сигналов, пересчет из милливольт в солнечные единицы потока.

Радиотелескоп РТ-3 - параллактическое поворотное устройство с электрическим приводом по часовому углу, на котором закреплено параболическое зеркало диаметром 3 м с совмещенными облучателями на длины волн 10.5 и 12 см (частоты 2.85 и 2.5 ГГц). Размещен в радиопрозрачном куполе диаметром 6 м для защиты от метеовоздействий, что существенно улучшает качество и стабильность данных. Ширина диаграммы направленности (ДН) составляет $2.3-2.5^{\circ}$. Это обеспечивает прием радиоизлучения от всей поверхности радиодиска Солнца, размер которого немного превышает $0.5^{\circ}$. Полоса пропускания каждого канала - 40 МГц, минимальная постоянная времени - 10 мс, запись сигнала осуществляется в цифровом виде с частотой отсчетов 1 Гц в дежурном режиме и 100 Гц в течение всплеска радиоизлучения. Проводятся ежедневные наблюдения в автоматическом режиме (без участия оператора) с передачей получаемых данных в Центральную базу данных для обеспечения оперативной диагностики солнечных вспышек.

Радиотелескоп РТ-2 - параллактическое поворотное устройство с электрическим приводом по часовому углу, на котором закреплено параболическое зеркало диаметром 2.5 м с совмещенными облучателями на длины волн 3 и 5 см (частоты 10 и 6 ГГц), размещен в радиопрозрачном куполе диаметром 6 м. Модуляционные радиометры прямого усиления построены на основе малошумящих сверхвысокочастотных усилителей. Аналого-цифровой преобразователь совместно с управляющим компьютером позволяет регистрировать в цифровом виде измеряемые сигналы с частотой отсчетов 1 Гц в дежурном режиме и 100 Гц в течение всплеска радиоизлучения в формате, пригодном для помещения в архив и для математической обработки результатов измерений. На длине волны 3 см ширина ДН составляет $1^{\circ} 15^{\prime}$, на длине волны 5 см - $1^{\circ} 45^{\prime}$. Полоса пропускания каждого канала 40 МГц, постоянная времени - 1 с. Проводятся ежедневные наблюдения в автоматическом режиме (без участия оператора) с передачей получаемых данных в Центральную базу данных для обеспечения оперативной диагностики солнечных вспышек.

Радиотелескоп РТ-М оборудован антенной размерами $4 \times 4$ м, которая является разновидностью синфазной решетки, состоящей из 160 диполей. Антенна имеет полосу пропускания от 250 до 350 МГц. Ширина ДН $-11^{\circ}$, полоса пропускания каждого канала - 300 кГц, минимальная постоянная времени - 1.25 мс, непрерывный динамический диапазон - 30 дБ, цифровая регистрация сигнала происходит с частотой 400 Гц. Для регистрации коронального излучения установлен радиоспектрограф Callisto, диапазон рабочих частот которого от 275 до 327 МГц (длины волн от 0.8 до 1.2 м). Проводятся ежедневные наблюдения в автоматическом режиме (без участия оператора) с передачей получаемых данных каждые 15 минут в Центральную базу данных для обеспечения оперативной диагностики солнечных вспышек (Вольвач и др., 2016).

Радиоастрономический диагностический комплекс Службы Солнца KRIM позволяет:

- получать ежедневный объем информации в широком диапазоне длин волн о событиях, которые происходят на Солнце и влияют на земные процессы;

- определять скорость распространения возмущения, генерируемую полосу частот, интенсивность энерговыделения, сведения о физических условиях в солнечной короне, момент прихода корпускулярного потока на Землю, интенсивность всплесков;

- проводить оценку ожидаемого масштаба геофизических возмущений. 
Результаты наблюдений заносятся в базу данных цифрового архива в виде двухбайтовых чисел типа integer. Каналы записываются по очереди, один на четных знакоместах файла, другой - на нечетных. Это обеспечивает взаимную привязку вариаций интенсивности по времени при любых манипуляциях с исходным файлом. Калибровочные данные располагаются на первых 10 знакоместах файла и автоматически считываются программой визуализации viewer.exе для оцифровки осей просматриваемого графика. Цифровой массив выбранного события (или его фрагмент) можно получить по адресу volvach@bk.ru.

\section{2 Наблюдения вспышечной активности в сентябре 2017 года}

6 сентября 2017 года в 12:02 UT радиотелескопом РT-M Службы Солнца KRIM была зафиксирована мощная вспышка X9.3 на частотах 275 и 327 МГц. Мощной вспышке предшествовала более слабая $\mathrm{X} 2.2$, она также была зафиксирована в сантиметровом диапазоне длин волн Службой Солнца KRIM радиотелескопами РT-2 и РТ-3. Эта вспышка произошла в той же области Солнца - NOAA 12673 , расположенной в гелиографических координатах S09 W45.

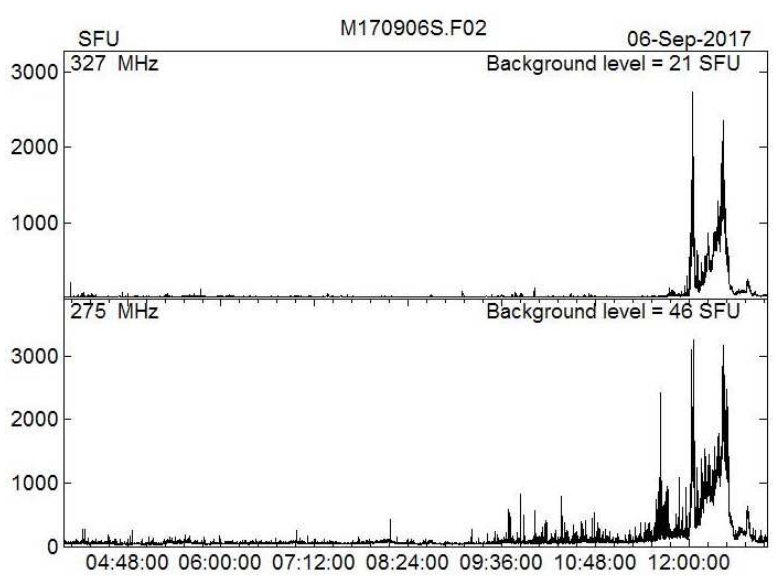

Рис. 2. Временные профили радиоизлучения на частотах 275 МГц (max $3260 \mathrm{SFU})$ и 327 МГц (max $2733 \mathrm{SFU})$

На рис. 2 представлены графики изменения интенсивности радиоизлучения во времени на частотах 275 и 327 МГц. На них хорошо отображены сильные радиовсплески (уровня R3), произошедшие в 12:02 UT с максимальным значением плотности спектрального потока 3260 SFU на частоте 275 МГц и 2733 SFU на частоте 327 МГц, зафиксированные радиотелескопом РТ-М. Для характеристики вспышечной активности Солнца помимо радиоизлучения удобно использовать величину потока рентгеновского излучения, так как во время вспышки его интенсивность может возрасти на несколько порядков. Особенно хорошо с потоком рентгеновского излучения согласуется радиоизлучение на длине волны в 10.7 см. В Службе Солнца KRIM для этого используется радиотелескоп РТ-3 с совмещенными облучателями на длины волн 10.5 и 12 см.

6 сентября 2017 г. спутники GOES 15 (Геостационарный оперативный спутник для изучения окружающего пространства) и GOES 14 также зафиксировали мощную рентгеновскую вспышку (восьмую по силе за последние 20 лет). Ей присвоен рентгеновский класс X9.3 и R3 - сильная по классификации мощности радиоизлучения. По данным Центра предсказания космической погоды (SWPC) максимальный уровень радиоизлучения вспышки соответствовал 14000 SFU на частоте 2650 МГц и $3200 \mathrm{SFU}$ на частоте 245 МГц, радиовсплеск II типа, генерируемый на фронте ударной волны, распространяющийся со скоростью ударной волны $1.765 \mathrm{~km} / \mathrm{c}$, и последующим радиовсплеском IV типа. GOES с 08:57 до 09:17 UT зафиксирована вспышка рентгеновского класса X2.2, а в 09:10 в 

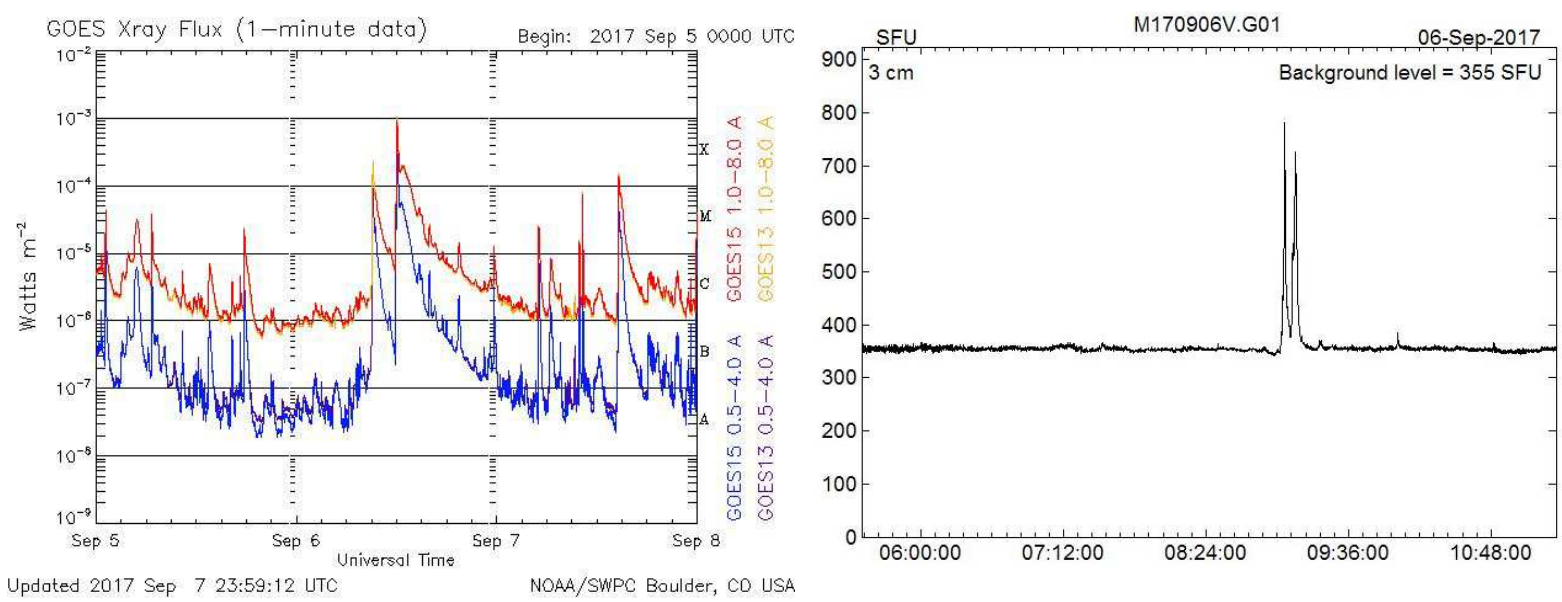

Рис. 3. Временной профиль рентгеновского излучения, измеренный GOES

Рис. 4. Временной профиль радиоизлучения на частоте 10 ГГц (max 691SFU)



Рис. 5. Временные профили радиоизлучения на частотах 2.5 ГГц (941 SFU) и 2.85 ГГц (578 SFU)

радиодиапазоне на длине волны в 10.7 см была зафиксирована вспышка с максимальным значением плотности спектрального потока в 410 SFU. Радиообсерватории Learmonth (Австралия) и San-Vito (Италия), входящие в сеть солнечных радиотелескопов (RSTN), принадлежащую Метеорологической службе ВВС США, также зафиксировали эти всплески на частотах 2695, 4995, 8800 и 15400 МГц ${ }^{1}$.

На рис. 3 приведено значение рентгеновского излучения, измеренного спутниками GOES, на котором четко видно наличие повышения интенсивности в двух местах, характеризующее две вспышки, которым присвоены классы Х2.2 (первой вспышке мощностью более $10^{-4} \mathrm{мBT} / \mathrm{m}^{2}$ ) и Х9.3 (второй вспышке мощностью $10^{-3}$ мВт/м ${ }^{2}$ ) в 12:02 UT, совпадающие по времени с зафиксированными Службой Солнца KRIM радиовсплесками.

На рис. 4 представлен график, на котором изображено изменение интенсивности радиоизлучения во времени на частоте 10 ГГц. На графике хорошо виден сильный радиовсплеск, произошедший в 09:10 UT с максимальным уровнем плотности спектрального потока $691 \mathrm{SFU}$, зафиксированный радиотелескопом РТ-2.

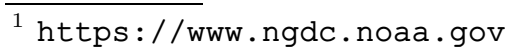


На рис. 5 представлены графики, на которых изображено изменение интенсивности радиоизлучения во времени на частотах 2.5 и 2.85 ГГц. На графиках хорошо виден сильный радиовсплеск, произошедший в 09:10 UT с максимальным уровнем плотности спектрального потока $941 \mathrm{SFU}$ на частоте 2.5 ГГц и $578 \mathrm{SFU}$ на частоте 2.85 ГГц, зафиксированный радиотелескопом РТ-3.

\section{3 Заключение}

Радиотелескопы Службы Солнца обеспечивают ежедневный мониторинг “космической погоды” в окрестностях Земли. Радиоастрономический диагностический комплекс Службы Солнца KRIM позволяет: получать ежедневный объем информации в широком диапазоне длин волн о событиях, которые происходят на Солнце и влияют на земные процессы; определять скорость распространения возмущения, генерируемую полосу частот, интенсивность энерговыделения, сведения о физических условиях в солнечной короне, момент прихода корпускулярного потока на Землю, интенсивность всплесков; проводить оценку ожидаемого масштаба геофизических возмущений. Полученная с помощью радиотелескопов Службы Солнца KRIM информация о радиовсплесках хорошо коррелирует с данными других наземных и космических обсерваторий, таких как RSTN и GOES, что подтверждает высокое качество регистрируемых Службой Солнца KRIM данных.

\section{Литература}

Вольвач А.Е., Юровский Ю.Ф., Самисько К.В. и др. // Изв. Крымск. Астрофиз. Обсерв. 2016. Т. 112. № 2. C. 179 .

Юровский Ю.Ф. // Кинем. и физ. небесн. тел. 2013. Т. 29. № 6. С. 68. 